Journal of Advanced Research in Fluid Mechanics and Thermal Sciences

Journal homepage: www.akademiabaru.com/arfmts.html ISSN: 2289-7879

\title{
New Insight into Gyrotactic Microorganisms in Anti-Infection Agents Through Wavy Deformable Catheter/Endoscope with Non-Linear Thermal Radiation: Numerical Study
}

\author{
Kh. S. Mekheimer ${ }^{1, *}$, Shaimaa Fathey Ramadan ${ }^{2, *}$ \\ Department of Mathematics, Faculty of Science (Boys), Al-Azhar University, Nasr-City,11884, Cairo, Egypt \\ Department of Mathematics, Faculty of Science (Girls), Al-Azhar University, Nasr-City, Cairo, Egypt
}

\section{ARTICLE INFO}

Article history:

Received 8 May 2020

Received in revised form 8 June 2020

Accepted 8 June 2020

Available online 20 September 2020

\section{Keywords:}

Peristaltic flow; Jeffrey Fluid; Nanoparticles; Microorganisms; Endoscope; Thermal Radiation

\section{ABSTRACT}

The reason for this work is to assess the impact of the peristaltic flow of Jeffery nanofluid containing motile gyrotactic microorganisms. This problem can be considered a numerical depiction of the fluid move through an endoscope or catheter tube. Where the catheter is covered with a blend of anti-infection agents and nanoparticles to oppose the formation of vital membranes. The effects of non-linear thermal radiation, viscous dissipation, and magnetic field are taken into consideration. Both inward and the external cylinders have sinusoidal waves traveling along their walls since there is a coupling between the occlusion of the external cylinder and the radius ratio. For long wavelength and low Reynolds number, a numerical study by using the Rung-Kutta-Merson method with Newton iteration in a shooting and matching technique is performed. The effect of physical implanted parameters is represented through a lot of charts for velocity, temperature, nanoparticle concentration, the density of motile microorganisms, shear stress, pressure rise, and pressure gradient.

\section{Introduction}

Nanoparticles have remarkable physicochemical, optical, and organic properties which can be controlled appropriately for wanted applications. Because of their little size, nanoparticles can enter the cell is inside microscopic organisms, parasites, infections, plant, and creature cells. Nanotechnology will get noteworthy advances in the determination and treatment of sickness. Foreseen applications in prescription incorporate medication conveyance in both in vitro and in vivo diagnostics, pharmaceuticals, and generation of improved biocompatible materials. The

* Corresponding author.

E-mail address: kh_mekheimer@azhar.edu.eg

* Corresponding author.

E-mail address: shaimaafathey2012@azhar.edu.eg

https://doi.org/10.37934/arfmts.75.3.2542 
nanomaterials in the antimicrobial application may evade the downsides of anti-toxins or help to improve their antimicrobial impact against the opposition of microorganisms. The progression of non-Newtonian liquid containing nanoparticles and microorganisms assume a significant job in the human body where nanoparticles appropriate for antibacterial covering on intravascular catheters. Mekheimer [1] explored the long-wavelength peristaltic transport through a uniform and nonuniform Annulus. Akbar and Nadeem [2] investigated the peristaltic flow of a micropolar fluid with nanoparticles in the small intestine. Nadeem et al., [3] analyzed the peristaltic flow of Walter's B fluid in the endoscope. Khan et al., [4] reported the mixed convection in gravity-driven thin-film nonNewtonian nanofluids to flow with gyrotactic microorganisms. Raju et al., [5] investigated the radiative flow of Casson fluid over a moving wedge filled with gyrotactic microorganisms.

In a living body, peristaltic flow happens because of synchronous constriction and development of smooth muscles that move over the entire length of a cylinder or channel. This instrument might be engaged with pee transport from the kidney to bladder through the ureter, food mixing and chime movement in the intestine, development of eggs in the Fallopian tube, the vehicle of the spermatozoa in the cervical trench, transport of bile in the bile pipe, transport of cilia, and flow of blood in little veins. The investigation of peristaltic flow with the impact of magnetic nanoparticles has various applications in biomedical engineering and material science. The nearness of the nanoparticles improves the electrical conductivity property of nanofluids, thus making them progressively defenseless to the impact of the magnetic field when contrasted with the regular base liquids. In the field of nanomedicine, magnetic nanoparticles are legitimately transmitted into a human body utilizing intravenous infusion technique. Bhatti et al., [6] discussed the simultaneous effects of coagulation and variable magnetic field on peristaltically induced motion of Jeffrey nanofluid containing gyrotactic microorganism. Mekheimer et al., [7] presented, Particulate suspension flow induced by sinusoidal peristaltic waves through eccentric cylinders. Bhatti and Zeeshan [8] examined the study of the variable magnetic field and endoscope on the peristaltic blood Flow of particle-fluid suspension through an annulus. Abdelsalam [9] studied the impact of impinging TiO2 nanoparticles in PrandtI nanofluid along with variable peristaltic blood flow. Zeeshan et al., [10] considered the mathematical study of peristaltic propulsion of solid-liquid multiphase flow with a rheological fluid as the base fluid in a duct. Mekheimer and elmaboud [11] illustrated the influence of heat transfer and magnetic field on peristaltic transport of a Newtonian fluid in a vertical annulus: Application of an endoscope. Hayat et al., [12] investigated the endoscopy and homogeneousheterogeneous reactions in MHD radiative peristaltic activity of Ree-Eyring fluid. Eldabe et al., [13] investigated the analytical, and numerical treatment to study the effects of Hall currents with viscous dissipation, heat absorption and chemical reaction on the peristaltic flow of Carreau nanofluid. Ellahi and Hussian [14] discussed the simultaneous effects of MHD and partial slip on the peristaltic flow of Jeffery fluid in a rectangular duct. Peristaltic transport of a particle-fluid suspension through a uniform and non-uniform annulus has been discussed by Mekheimer and elmaboud [15]. Hasona et al., [16] illustrated the combined effects of magnetohydrodynamic and temperature-dependent viscosity on the peristaltic flow of Jeffrey nanofluid through a porous medium: Applications to oil refinement. Rachid and Ouazzani [17] studied the electro-magnetohydrodynamic peristaltic pumping of a bioviscosity fluid between two coaxial deformable tubes through a porous medium. Magnetohydrodynamic (MHD) slip Darcy flow of viscoelastic fluid over a stretching sheet and heat transfer with thermal radiation and viscous dissipation has been investigated by Wahid et al., [18]. Peristaltic motion of Eyring-Powell nano fluid with couple stresses and heat and mass transfer through a porous media under the effect of magnetic field inside asymmetric vertical channel has been discussed by Eldabe et al., [19]. 
With the potential applications and past examinations, we have exhibited the peristaltic flow of Jeffrey nanofluid containing motile gyrotactic microorganisms through an endoscope under the impact of non-linear thermal radiation and magnetic field. The system of partial differential equations which portray this phenomenon with subjected boundary conditions have been modulated under long wavelength and low Reynolds number suppositions and solved numerically by utilizing Rung-Kutta-Merson strategy with Newton emphasis in a shooting and matching procedure. The physical practices of rising parameters for velocity, temperature, the density of motile microorganisms, shear stress, pressure rise, and pressure gradient are discussed through a set of graphs.

\section{Methodology}

The peristaltic transport of MHD Jeffrey Nanofluid containing motile gyrotactic microorganisms is considered between the internal holes between both cylinders. Both the internal and the external cylinders have sinusoidal waves traveling down their walls. Cylindrical polar coordinate system $(R, Z)$ is chosen, where $R$ is taken along the radial direction and $z$-axis is taken along the axis of tube. An external magnetic field is applied without an electric field, the incited magnetic is dismissed because of little magnetic Reynold's number. Figure 1 demonstrated the physical model of the problem.

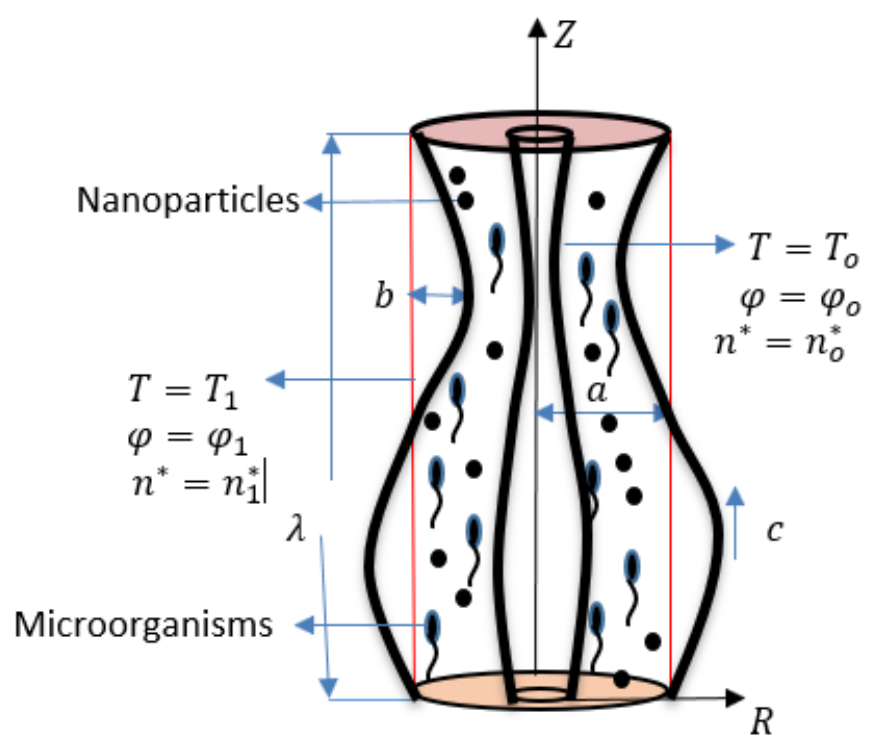

Fig. 1. Geometry of the physical model

The geometry of the walls surface in the fixed frame $(R, Z)$ are described as

$$
\begin{aligned}
& R_{2}=a+b \sin \frac{2 \pi}{\lambda}(Z-c t), \\
& R_{1}=n R_{2}=n a+n b \sin \frac{2 \pi}{\lambda}(Z-c t), \quad 0<n<1,
\end{aligned}
$$

We introduce a wave frame of reference $(r, z)$ moving with velocity $c$ in which the motion becomes independent of time when the channel length is an integral multiple of the wavelength. The transformation from the fixed frame of reference $(R, Z)$ to the wave frame of reference $(r, z)$ is given by 
$r=R, \quad z=Z-c t$, and $p=P$,

$u(r, z)=U(R, Z-c t), \mathrm{w}(\mathrm{r}, \mathrm{z})=W(R, Z-c t)-c$,

The uniform magnetic field $B$ is applied in the radial direction as follows

$B=\left(B_{o}, 0,0\right)$,

The Ohms law with no electric field is

$J=\sigma(V \times B)$

$J \times B=\left(0,0,-\sigma B_{o}^{2} w\right)$,

The relevant equations governing the fluid flow in the wave frame are

$\frac{\partial u}{\partial r}+\frac{u}{r}+\frac{\partial w}{\partial z}=0$

$\rho_{f}\left[u \frac{\partial u}{\partial r}+w \frac{\partial u}{\partial z}\right]=-\frac{\partial p}{\partial r}+\frac{1}{r} \frac{\partial}{\partial r}\left(r \tau_{r r}\right)+\frac{\partial \tau_{r z}}{\partial z}$

$\rho_{f}\left[u \frac{\partial w}{\partial r}+w \frac{\partial w}{\partial z}\right]=-\frac{\partial p}{\partial z}+\frac{1}{r} \frac{\partial}{\partial r}\left(r \tau_{r z}\right)+\frac{\partial \tau_{z z}}{\partial z}-\sigma B_{o}^{2} w+\rho_{f} g \beta\left(1-\varphi_{1}\right)\left(T-T_{1}\right)-g\left(\rho_{p}-\rho_{f}\right)(\varphi-$ $\left.\varphi_{1}\right)-g \gamma\left(\rho_{m}-\rho_{f}\right)\left(n^{*}-n_{1}^{*}\right)$

$(\rho c)_{f}\left(u \frac{\partial T}{\partial r}+w \frac{\partial T}{\partial z}\right)=k_{T}\left(\frac{\partial^{2} T}{\partial r^{2}}+\frac{1}{r} \frac{\partial T}{\partial r}+\frac{\partial^{2} T}{\partial Z^{2}}\right)+(\rho c)_{p}\left(D_{B}\left(\frac{\partial \varphi}{\partial r} \frac{\partial T}{\partial r}+\frac{\partial \varphi}{\partial z} \frac{\partial T}{\partial z}\right)+\frac{D_{T}}{T_{o}}\left(\left(\frac{\partial T}{\partial r}\right)^{2}+\right.\right.$

$\left.\left.\left(\frac{\partial T}{\partial z}\right)^{2}\right)\right)+\left(\tau_{r r} \frac{\partial u}{\partial r}+\tau_{r z}\left(\frac{\partial u}{\partial z}+\frac{\partial w}{\partial r}\right)+\tau_{z z} \frac{\partial w}{\partial z}\right)+\sigma B_{o}^{2} w^{2}-\frac{\partial q_{r}}{\partial r}$

$\left(u \frac{\partial \varphi}{\partial r}+w \frac{\partial \varphi}{\partial z}\right)=D_{B}\left(\frac{\partial^{2} \varphi}{\partial r^{2}}+\frac{1}{r} \frac{\partial \varphi}{\partial r}+\frac{\partial^{2} \varphi}{\partial z^{2}}\right)+\frac{D_{T}}{T_{o}}\left(\frac{\partial^{2} T}{\partial r^{2}}+\frac{1}{r} \frac{\partial T}{\partial r}+\frac{\partial^{2} T}{\partial Z^{2}}\right)$

$u \frac{\partial n^{*}}{\partial r}+w \frac{\partial n^{*}}{\partial z}+\frac{b^{*} W_{c}}{\left(\varphi_{o}-\varphi_{1}\right)}\left(\frac{\partial}{\partial r}\left(n^{*} \frac{\partial \varphi}{\partial r}\right)+\frac{\partial}{\partial z}\left(n^{*} \frac{\partial \varphi}{\partial z}\right)\right)=D_{m}\left(\frac{\partial^{2} n^{*}}{\partial r^{2}}+\frac{1}{r} \frac{\partial n^{*}}{\partial r}+\frac{\partial^{2} n^{*}}{\partial z^{2}}\right)$

The appropriate boundary conditions are given as follows

$w=-c, T=T_{o}, \varphi=\varphi_{o}, n^{*}=n_{o}^{*}$ at $r=r_{1}=n r_{2}$,

$w=-c, T=T_{1}, \varphi=\varphi_{1}, n^{*}=n_{1}^{*}$ at $r=r_{2}=a+b \sin \left(\frac{2 \pi z}{\lambda}\right)$

The stress tensor $\tau$ for incompressible Jeffrey fluid [13] can be expressed as

$\tau=\frac{\mu}{1+\lambda_{1}}\left[\dot{\gamma}+\lambda_{2} \ddot{\gamma}\right]$

In view of Rosseland approximation, nonlinear radiative heat flux $q_{r}$ can be written as 
$q_{r}=-\frac{4 \sigma^{*}}{3 k^{*}} \frac{\partial T^{4}}{\partial r}$

Let us introduce the following dimensionless quantities as

$r^{*}=\frac{r}{a}, z^{*}=\frac{z}{\lambda}, u^{*}=\frac{\lambda u}{a c}, w^{*}=\frac{w}{c}, t^{*}=\frac{t c}{\lambda}, p^{*}=\frac{a^{2} p}{\lambda \mu c}, \delta=\frac{a}{\lambda}, \tau_{i j}^{*}=\frac{\tau_{i j} a}{\mu c}, r_{1}^{*}=\frac{r_{1}}{a}, r_{2}^{*}=\frac{r_{2}}{a}$,

$\phi_{1}=\frac{b}{a}, \theta=\frac{T-T_{1}}{T_{o}-T_{1}}, \varphi^{*}=\frac{\varphi-\varphi_{1}}{\varphi_{o}-\varphi_{1}}, \chi=\frac{n^{*}-n_{1}^{*}}{n_{o}^{*}-n_{1}^{*}}$

After substituting from Eq. (16), Eq. (7)-(13) can be written in dimensionless form after dropping the star mark as

$\frac{\partial u}{\partial r}+\frac{u}{r}+\frac{\partial w}{\partial z}=0$

$\operatorname{Re} \delta^{3}\left[u \frac{\partial u}{\partial r}+w \frac{\partial u}{\partial z}\right]=-\frac{\partial p}{\partial r}+\frac{\delta}{r} \frac{\partial}{\partial r}\left(r \tau_{r r}\right)+\delta^{2} \frac{\partial \tau_{r z}}{\partial z}$

$\operatorname{Re} \delta\left[u \frac{\partial w}{\partial r}+w \frac{\partial w}{\partial z}\right]=-\frac{\partial p}{\partial z}+\frac{1}{r} \frac{\partial}{\partial r}\left(r \tau_{r z}\right)+\delta \frac{\partial \tau_{z z}}{\partial z}-M^{2} w+G_{r}\left(\theta-N_{r} \varphi-R_{b} \chi\right)$

$\operatorname{Re} P_{r} \delta\left[u \frac{\partial \theta}{\partial r}+w \frac{\partial \theta}{\partial z}\right]=\left(\frac{\partial^{2} \theta}{\partial r^{2}}+\frac{1}{r} \frac{\partial \theta}{\partial r}+\delta^{2} \frac{\partial^{2} \theta}{\partial z^{2}}\right)+N_{b}\left(\frac{\partial \varphi}{\partial r} \frac{\partial \theta}{\partial r}+\delta^{2} \frac{\partial \varphi}{\partial z} \frac{\partial \theta}{\partial z}\right)+N_{t}\left(\left(\frac{\partial \theta}{\partial r}\right)^{2}+\delta^{2}\left(\frac{\partial \theta}{\partial z}\right)^{2}\right)+$ $E_{c} P_{r}\left(\delta \tau_{r r} \frac{\partial u}{\partial r}+\tau_{r z}\left(\delta^{2} \frac{\partial u}{\partial z}+\frac{\partial w}{\partial r}\right)+\delta \tau_{z z} \frac{\partial w}{\partial z}\right)+M^{2} E_{c} P_{r} w^{2}+R_{d}\left(3\left(1+\left(\theta_{1}-1\right) \theta\right)^{2}\left(\theta_{1}-\right.\right.$

1) $\left.\left(\frac{\partial \theta}{\partial r}\right)^{2}+\left(1+\left(\theta_{1}-1\right) \theta\right)^{3}\left(\theta_{1}-1\right) \frac{\partial^{2} \theta}{\partial r^{2}}\right)$

$\operatorname{Re} L_{e} \delta\left[u \frac{\partial \varphi}{\partial r}+w \frac{\partial \varphi}{\partial z}\right]=\left(\frac{\partial^{2} \varphi}{\partial r^{2}}+\frac{1}{r} \frac{\partial \varphi}{\partial r}+\delta^{2} \frac{\partial^{2} \varphi}{\partial z^{2}}\right)+\frac{N_{t}}{N_{b}}\left(\frac{\partial^{2} T}{\partial r^{2}}+\frac{1}{r} \frac{\partial T}{\partial r}+\delta^{2} \frac{\partial^{2} T}{\partial Z^{2}}\right)$

$\operatorname{Re} L_{b} \delta\left[u \frac{\partial \chi}{\partial r}+w \frac{\partial \chi}{\partial z}\right]+P_{e}\left(\frac{\partial}{\partial r}\left(\chi \frac{\partial \varphi}{\partial r}\right)+\delta^{2} \frac{\partial}{\partial z}\left(\chi \frac{\partial \varphi}{\partial z}\right)\right)=\left(\frac{\partial^{2} \chi}{\partial r^{2}}+\frac{1}{r} \frac{\partial \chi}{\partial r}+\delta^{2} \frac{\partial^{2} \chi}{\partial z^{2}}\right)$

The dimensionless boundary conditions are

$w=-1, \theta=1, \varphi=1, \chi=1$ at $r=r_{1}=n r_{2}$,

$w=-1, \theta=0, \varphi=0, \chi=0$ at $r=r_{2}=1+\phi_{1} \sin (2 \pi z)$

For long wavelength (i.e, $\delta \ll 1$ ) and low Reynolds number (i.e., Re $\rightarrow 0$ ) the system of our Eq. (18)-(22) can be reduced to

$\frac{\partial p}{\partial r}=0$

$\frac{1}{1+\lambda_{1}} \frac{1}{r} \frac{\partial}{\partial r}\left(r \frac{\partial w}{\partial r}\right)-M^{2} w+G_{r}\left(\theta-N_{r} \varphi-R_{b} \chi\right)=\frac{\partial p}{\partial z}$

$\frac{1}{r} \frac{\partial}{\partial r}\left(r \frac{\partial \theta}{\partial r}\right)+N_{b} \frac{\partial \varphi}{\partial r} \frac{\partial \theta}{\partial r}+N_{t}\left(\frac{\partial \theta}{\partial r}\right)^{2}+\frac{E_{c} P_{r}}{1+\lambda_{1}}\left(\frac{\partial w}{\partial r}\right)^{2}+M^{2} E_{c} P_{r} w^{2}+R_{d}\left(3\left(1+\left(\theta_{1}-1\right) \theta\right)^{2}\left(\theta_{1}-\right.\right.$

1) $\left.\left(\frac{\partial \theta}{\partial r}\right)^{2}+\left(1+\left(\theta_{1}-1\right) \theta\right)^{3}\left(\theta_{1}-1\right) \frac{\partial^{2} \theta}{\partial r^{2}}\right)=0$ 
$\frac{1}{r} \frac{\partial}{\partial r}\left(r \frac{\partial \varphi}{\partial r}\right)+\frac{N_{t}}{N_{b}} \frac{1}{r} \frac{\partial}{\partial r}\left(r \frac{\partial \theta}{\partial r}\right)=0$

$\frac{1}{r} \frac{\partial}{\partial r}\left(r \frac{\partial \chi}{\partial r}\right)-P_{e} \frac{\partial}{\partial r}\left(\chi \frac{\partial \varphi}{\partial r}\right)=0$

The parameters are defined as

$$
\begin{aligned}
& M^{2}=\frac{\sigma B_{o}^{2} a^{2}}{\mu}, G_{r}=\frac{g \beta\left(1-\varphi_{1}\right)\left(T_{o}-T_{1}\right)}{a c}, N_{r}=\frac{\left(\rho_{p}-\rho_{f}\right)\left(\varphi_{o}-\varphi_{1}\right)}{\beta \rho_{f}\left(1-\varphi_{1}\right)\left(T_{o}-T_{1}\right)}, R_{b}=\frac{\gamma\left(\rho_{m}-\rho_{f}\right)\left(n_{o}^{*}-n_{1}^{*}\right)}{\beta \rho_{f}\left(1-\varphi_{1}\right)\left(T_{o}-T_{1}\right)}, P_{r}= \\
& \frac{v(\rho c)_{f}}{k_{T}}, N_{b}=\frac{(\rho c)_{p} D_{B}\left(\varphi_{o}-\varphi_{1}\right)}{k_{T}}, N_{t}=\frac{(\rho c)_{p} D_{T}\left(T_{o}-T_{1}\right)}{k_{T} T_{1}}, R_{d}=\frac{16 \sigma^{*} T_{1}^{3}}{3 k_{T} k^{*}}, \theta_{1}=\frac{T_{o}}{T_{1}}, E_{c}=\frac{c^{2}}{c_{f}\left(T_{o}-T_{1}\right)}, \\
& L_{e}=\frac{v}{D_{B}}, \quad L_{b}=\frac{v}{D_{m}}, P_{e}=\frac{b^{*} W_{c}}{D_{m}},
\end{aligned}
$$

\section{Flow Rate, Pressure Rise, and Wall Shear Stress (WSS)}

The time-averaged flow rate is given by

$Q=\int_{0}^{1} Q(Z, t) d t=q+\left(1-n^{2}\right) r_{2}^{2}$

The volume flow rate in the wave frame $q$ calculate as follows

$q=2 \int_{r_{1}}^{r_{2}} w r d r$

The pressure rise across one wavelength in the non-dimensional form is given by

$$
\Delta p=\int_{0}^{1} \frac{d p}{d z} d z
$$

The shear stresses $\tau_{w}$ at the outer surface of the inner tube and the inner surface of the outer tube are given by

$$
\tau_{w 1}=\frac{1}{1+\lambda_{1}}\left(\frac{\partial w}{\partial r}\right)_{r=r_{1}} \quad, \quad \tau_{w 2}=\frac{1}{1+\lambda_{1}}\left(\frac{\partial w}{\partial r}\right)_{r=r_{2}}
$$

\section{Numerical Solution Method}

The system of our nonlinear ordinary differential equations is solved by the numerical method based on the Rung-Kutta-Merson method with Newton iteration in a shooting and matching technique. Use the following transformations

$$
w=Y_{1}, \quad \theta=Y_{3}, \quad \varphi=Y_{5}, \quad \chi=Y_{7}
$$

Eq. (24)-(28) with the boundary condition in Eq. (23) can be written as follows

$$
Y_{1}^{\prime}=Y_{2} \quad, Y_{2}^{\prime}=-\frac{1}{r} Y_{2}+M^{2} Y_{1}-G_{r}\left(Y_{3}-N_{r} Y_{5}-R_{b} Y_{7}\right)-p_{1}
$$




$$
\begin{aligned}
& Y_{3}^{\prime}=Y_{4}, \quad\left(1-R_{d}\left(1+\left(\theta_{1}-1\right) Y_{3}\right)^{3}\left(\theta_{1}-1\right)\right) Y_{4}^{\prime}=-\frac{1}{r} Y_{4}-N_{b} Y_{4} Y_{6}-N_{t}\left(Y_{4}\right)^{2}- \\
& \frac{1}{1+\lambda_{1}} E_{c} P_{r}\left(Y_{2}\right)^{2}-M^{2} E_{c} P_{r}\left(Y_{1}\right)^{2}+R_{d}\left(3\left(1+\left(\theta_{1}-1\right) Y_{3}\right)^{2}\left(\theta_{1}-1\right)\left(Y_{4}\right)^{2}\right), \\
& Y_{5}^{\prime}=Y_{6}, \quad Y_{6}^{\prime}=-\frac{1}{r} Y_{6}-\frac{N_{t}}{N_{b}}\left(Y_{2}^{\prime}+\frac{1}{r} Y_{2}\right) \\
& Y_{7}^{\prime}=Y_{8}, \quad Y_{8}^{\prime}=-\frac{1}{r} Y_{8}+P_{e}\left(Y_{6} Y_{8}+Y_{6}^{\prime} Y_{7}\right)
\end{aligned}
$$

Subject to the boundary conditions

$$
\begin{aligned}
& Y_{1}=-1, \quad Y_{3}=1, \quad Y_{5}=1 \quad \text { and } Y_{7}=1 \quad \text { at } r=r_{1}, \\
& Y_{1}=-1, \quad Y_{3}=0, \quad Y_{5}=0 \quad \text { and } Y_{7}=0 \quad \text { at } r=r_{2},
\end{aligned}
$$

where the prime denotes the differentiation with respect to $r$. To compute the physical quantities $w, \theta, \varphi$ and $\chi$. Mathematica package version 9 is used to solve the system of Eq. (34)-(37) with boundary conditions in Eq. (38) and (33).

\section{Graphical Results and Discussion}

This section examines the graphical outcomes for the different physical parameters associated with the peristaltic flow of Jeffery nanofluid containing motile gyrotactic microorganisms through an endoscope under the impact of non-linear thermal radiation and magnetic field. Consequently, a Mathematica program (Rung-Kutta-Merson method with Newton iteration in a shooting and matching technique) has been utilized to analyze the physical clarification of the included parameters in the flow field.

\subsection{Velocity Profiles}

The velocity $w$ of the flow field is found to change more or less with the flow parameters. The impact of the flow parameters on these distributions is investigated with the assistance of Figure 27.

Figure 2 show the impact of the non-Newtonian parameter $\lambda_{1}$ on the velocity on account of existing microorganisms and nanoparticles and in their nonappearances. The velocity is higher in the absence of microorganisms and nanoparticles. It is additionally seen that the most extreme velocity happens at the center of the separation between the two deformable cylinders. This outcome agrees with the physical expectation since exist microorganisms and nanoparticles that represent an obstacle to flow and therefore, reduced its velocity.

Figure 3 portrays the impact of the magnetic parameter $M$ and Grashof number $G_{r}$ on the velocity. One is to distinguish that the rising values of $M$ and $G_{r}$ augment the velocity. Figure 4 impacts the effect of $N_{r}$ and $R_{b}$ on velocity, it is noted that, an expansion in $N_{r}$ and $R_{b}$ attributes the fluid towards the surface thus diminishing the velocity. 


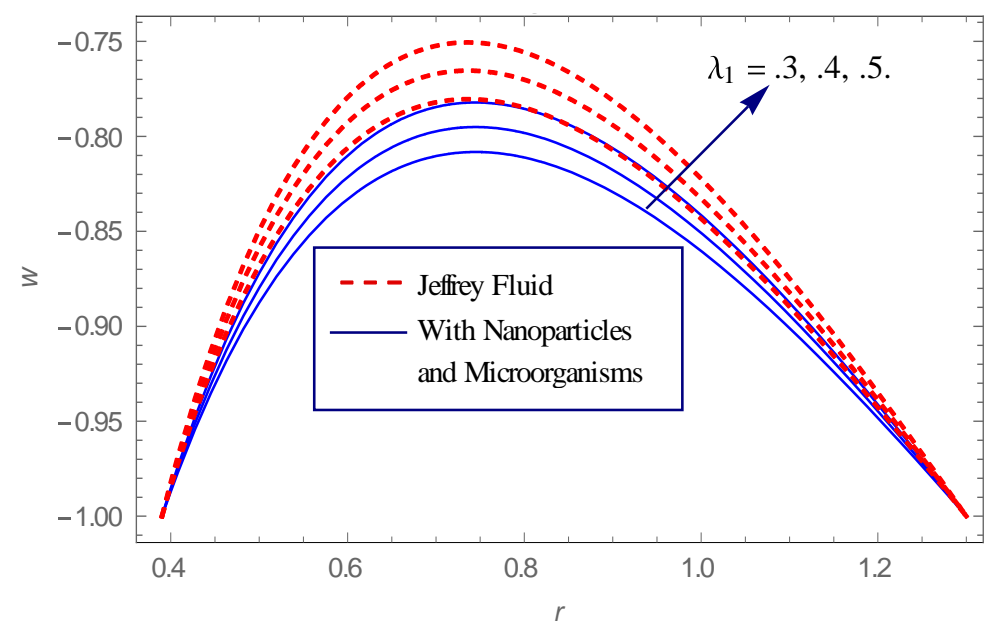

Fig. 2. The velocity of fluid particles $w$ is plotted against $r$, for $M=1, G_{r}=3, N_{r}=.1, R_{b}=.2, P_{1}=.5, P_{r}=.7, N_{t}=.1, N_{b}=$ .1, $E_{c}=.1, R_{d}=.4, \theta_{1}=1.1, P_{e}=1$

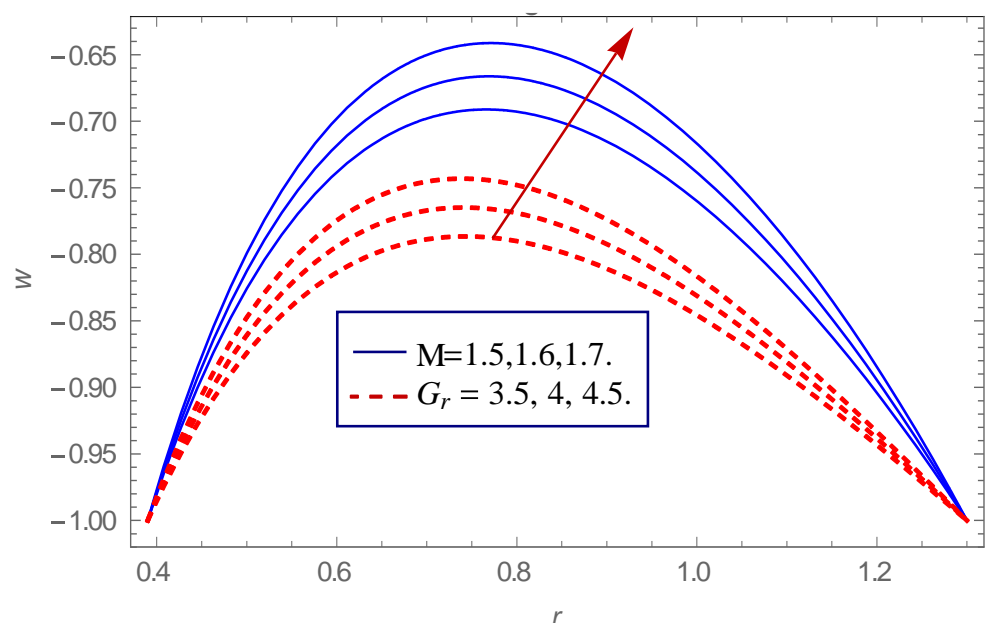

Fig. 3. The velocity of fluid particles $w$ is plotted against $r$, for $N_{t}=.1, N_{r}=.1, R_{b}=.2, \lambda_{1}=.3, P_{r}=.7, N_{b}=.1, E_{c}=$ $.1, R_{d}=.4, \theta_{1}=1.1, P_{e}=1$

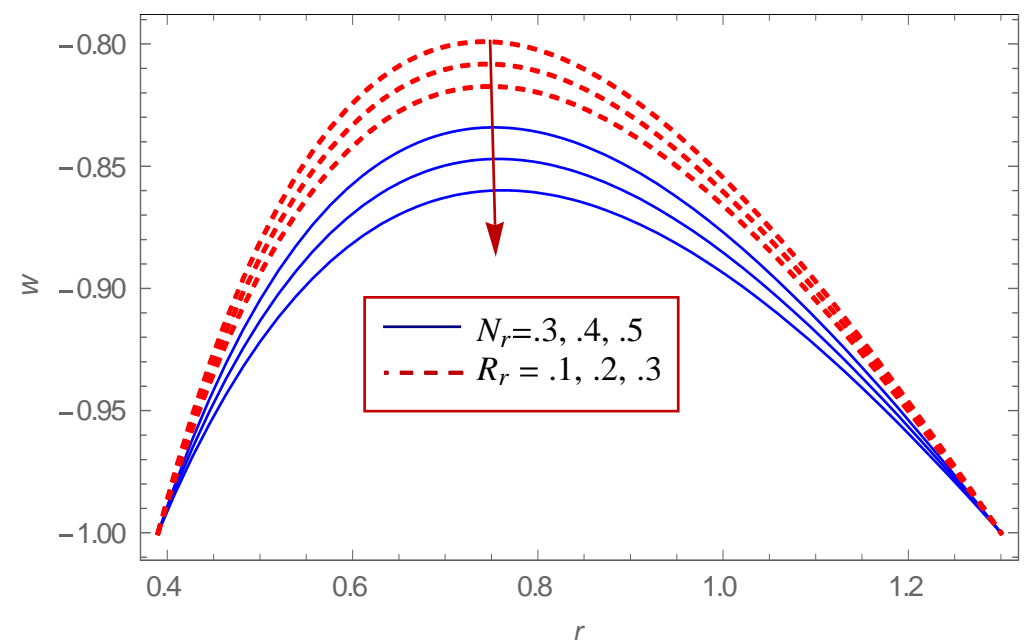

Fig. 4. The velocity of fluid particles $w$ is plotted against $r$, for $N_{t}=.1, G_{r}=3, M=1, \lambda_{1}=.3, P_{r}=.7, N_{b}=.1, E_{c}=.1, R_{d}=$ .4, $\theta_{1}=1.1, P_{e}=1$ 
Figure 5 is portrayed to visualize the variety in the velocity because of the impact of the Brownian motion parameter $N_{b}$ and the thermophoresis parameter $N_{t}$. One is to recognize that the rising values of $N_{b}$ and $N_{t}$ augment the velocity. Figure 6 delineate the impact of differing the span of the inward cylinder by changing $n$ on the velocity, plainly the speed diminishes with expanding $n$ close to the external surface of the internal cylinder, while the speed stays steady close to the inward surface of the external cylinder.

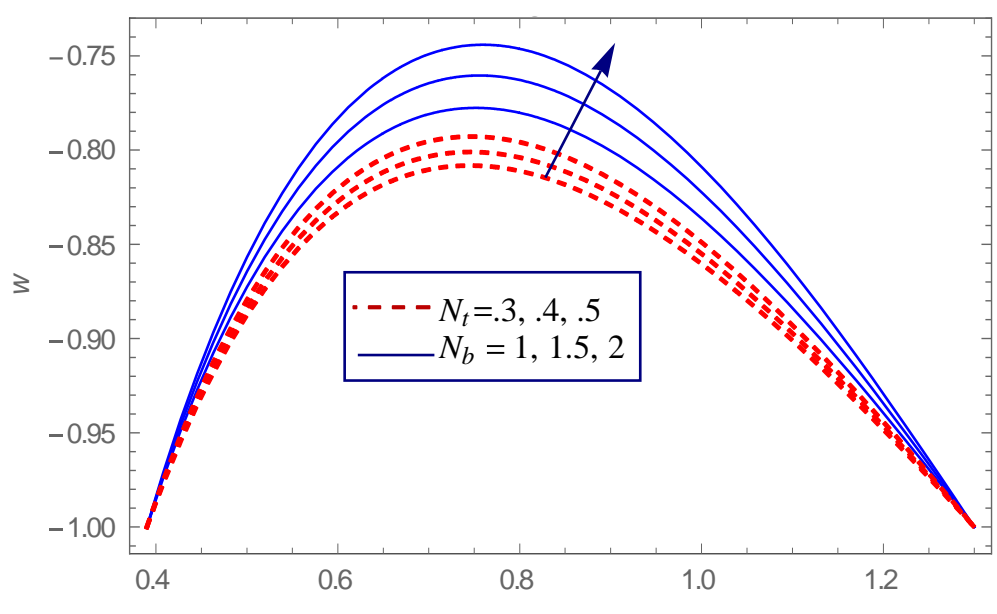

Fig. 5. The velocity of fluid particles $w$ is plotted against $r$, for $N_{b}=.1, G_{r}=3, M=1, \lambda_{1}=.3, P_{r}=.7, N_{t}=.1, E_{c}=.1, R_{d}=$ .4, $\theta_{1}=1.1, P_{e}=1$

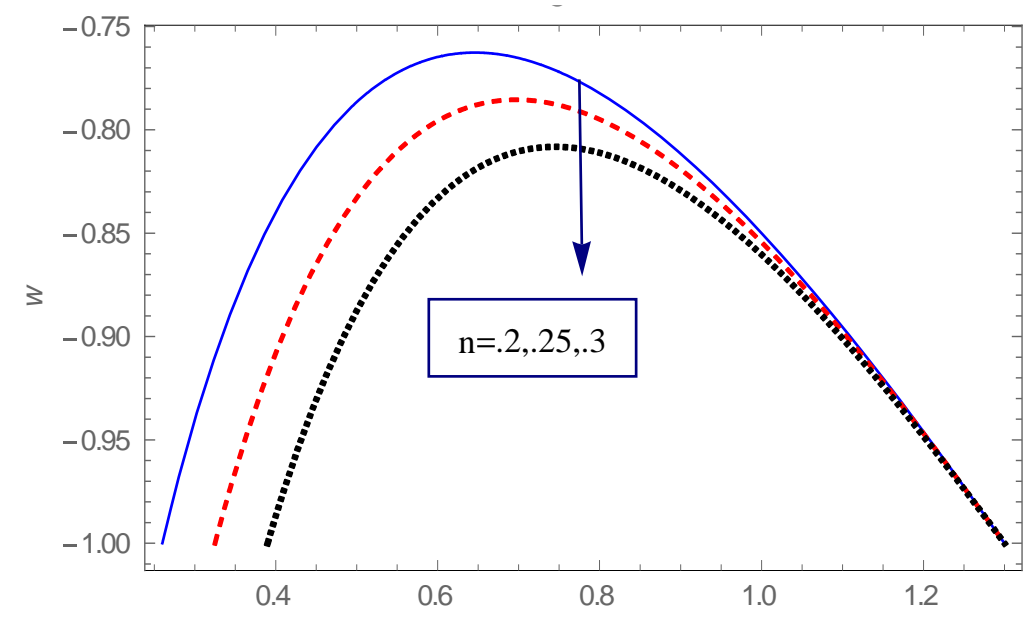

Fig. 6. The velocity of fluid particles $w$ is plotted against $r$, for $N_{t}=.1, N_{r}=.1, R_{b}=.2, \lambda_{1}=.3, G_{r}=3, P_{r}=.7, N_{b}=.1, E_{c}=$ $.1, R_{d}=.4, \theta_{1}=1.1, P_{e}=M=1$

Figure 7 is portrayed to show the velocity in the instances of deformable cylinders, the internal cylinder is solid and annulus. Plainly the velocity takes a higher quality when the inward cylinder is solid, while the velocity takes little qualities on account of the annulus. 


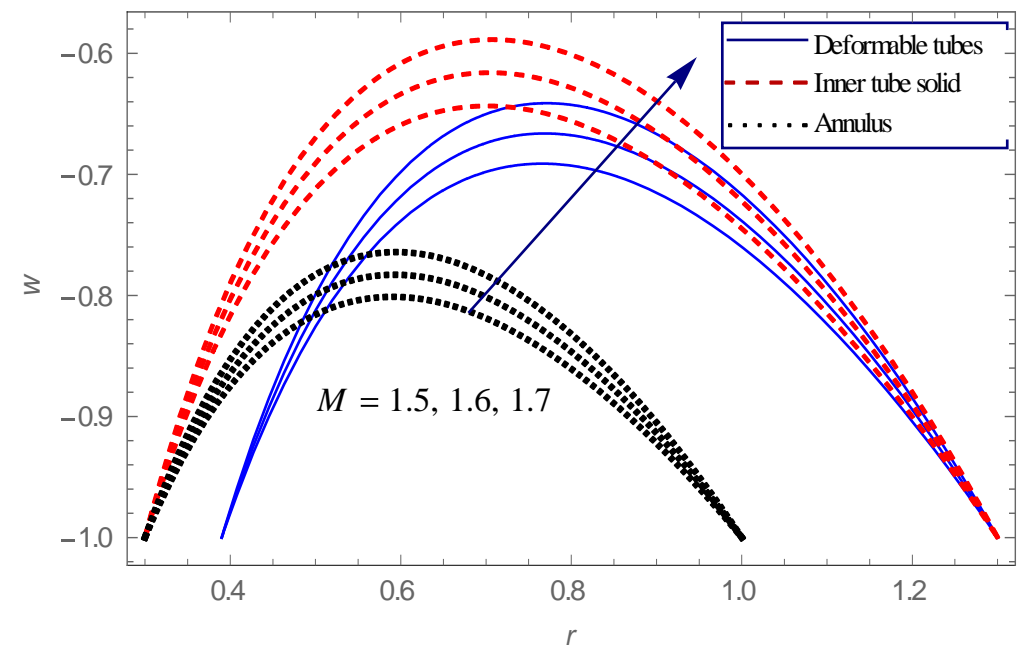

Fig. 7. The velocity of fluid particles $w$ is plotted against $r$, for $N_{b}=.1, E_{c}=.1, R_{d}=.4, \theta_{1}=1.1, N_{t}=.1, N_{r}=.1, R_{b}=.2$, $\lambda_{1}=.3, P_{r}=.7, P_{c}=1, G_{r}=3$

\subsection{Temperature Profiles}

Figure 8 is outlined to examine the impact of the temperature ratio parameter $\theta_{1}$ on the temperature for both Jeffery fluid and Jeffery fluid with nanoparticles and microorganisms. It is seen that $\theta_{1}$ prompts an intensifying in temperature for the two cases, yet the temperature is higher on account of the nearness of microorganisms and nanoparticles. Figure 9 translates the impacts of the Brownian motion parameter $N_{b}$, the thermophoresis parameter $N_{t}$, and non-linear radiation parameter $R_{d}$ on the temperature distribution. From this plot, we identified that the expansion in the values of Brownian motion, thermophoresis, and non-linear radiation parameters amplifies the temperature. This reliable with the physical concerned that the Brownian motion depends on the unsystematic movement of fluid particles on the surface and the rise of $N_{b}$ improves this motion of the fluid particles which causes much heat. Likewise, the expanding values of $N_{t}$ physically imply that the nanoparticles are moving far from the hot surface to cool which fluid temperature rise.

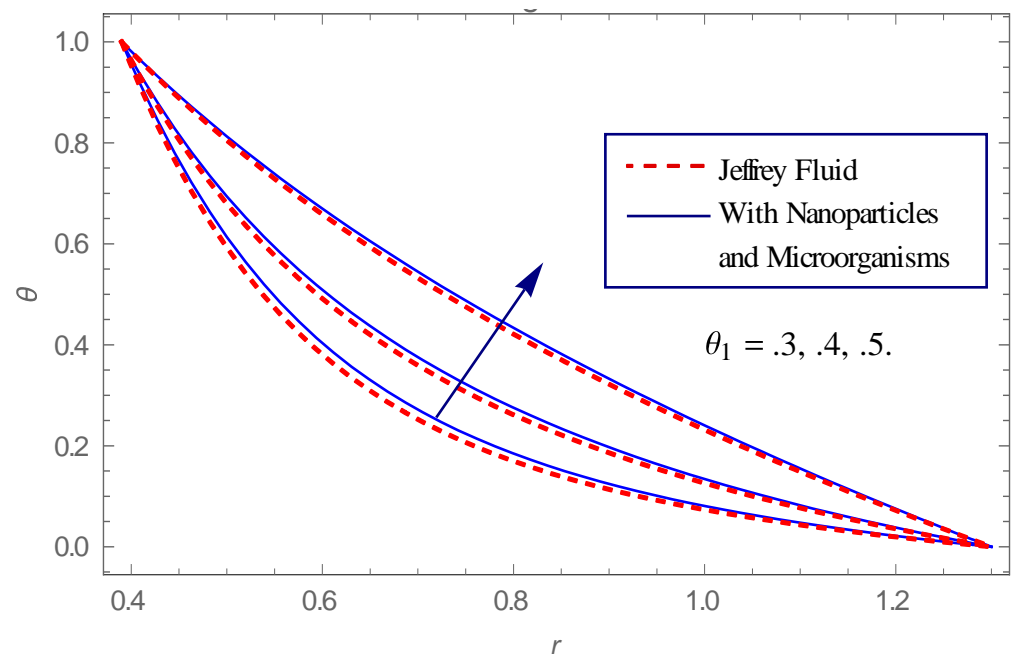

Fig. 8. The temperature of fluid particles $\theta$ is plotted against $r$, $M=1, G_{r}=3, N_{r}=.1, R_{b}=.2, P_{1}=.5, P_{r}=.7, N_{t}=.1, N_{b}=$ $.1, E_{c}=.1, R_{d}=1.2, \lambda_{1}=.3, P_{e}=1$ 


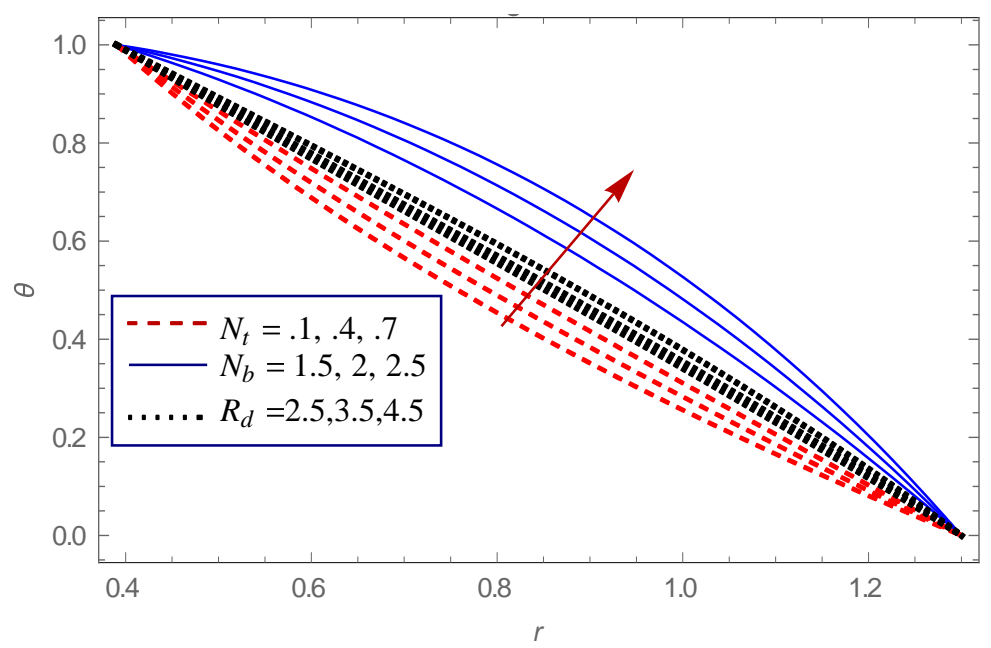

Fig. 9. The temperature of fluid particles $\theta$ is plotted against $r$, for $N_{r}=.1, G_{r}=3, M=1, \lambda_{1}=.3, P_{r}=.7, R_{b}=.2, E_{c}=$ $.1, R_{d}=.4, \theta_{1}=1.1, P_{e}=1$

\subsection{Nanoparticle Concentration Profiles}

Figure 10 is intended to research the effect of the thermophoresis parameter $N_{t}$ and the Brownian motion parameter $N_{b}$ on nanoparticle concentration. It can be seen from this plot that the concentration of the nanoparticles decay with the expansion of $N_{t}$ While the opposite impact happens by increasing $N_{b}$. Figure 11 delineate the nanoparticle's phenomena distribution for a few values of non-linear radiation parameter $R_{d}$ and the temperature ratio parameter $\theta_{1}$. The nanoparticle's phenomena decline as the non-linear radiation and the temperature ratio increment.

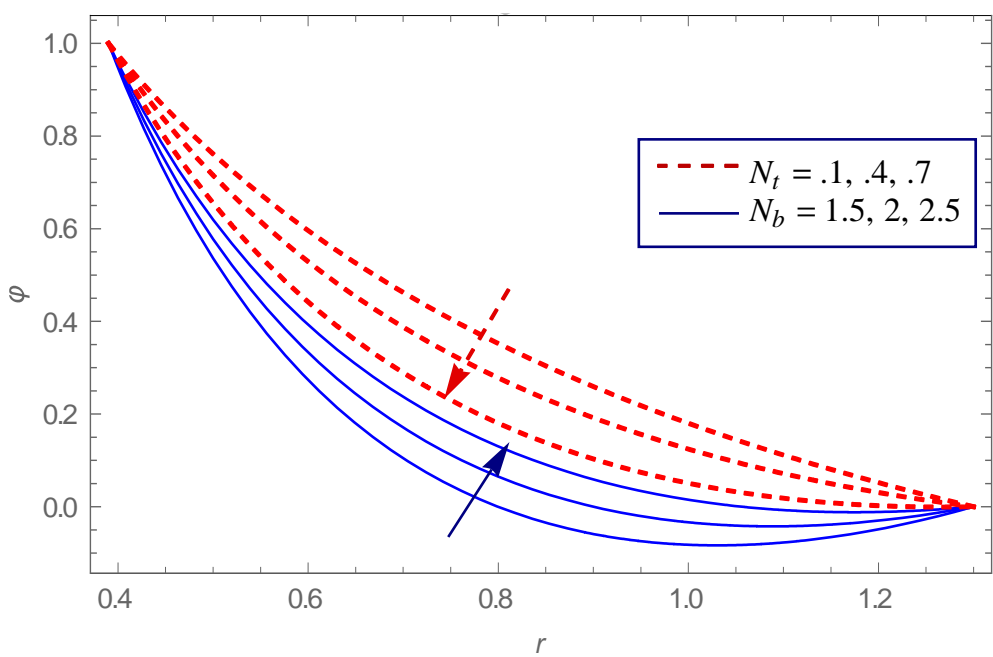

Fig. 10. The nanoparticles concentration of fluid particles $\varphi$ is plotted against $r$, for $N_{r}=.1, G_{r}=3, M=1, \lambda_{1}=.3, P_{r}=$ $.7, R_{b}=.2, E_{c}=.1, R_{d}=.4, \theta_{1}=1.1, P_{e}=1$ 


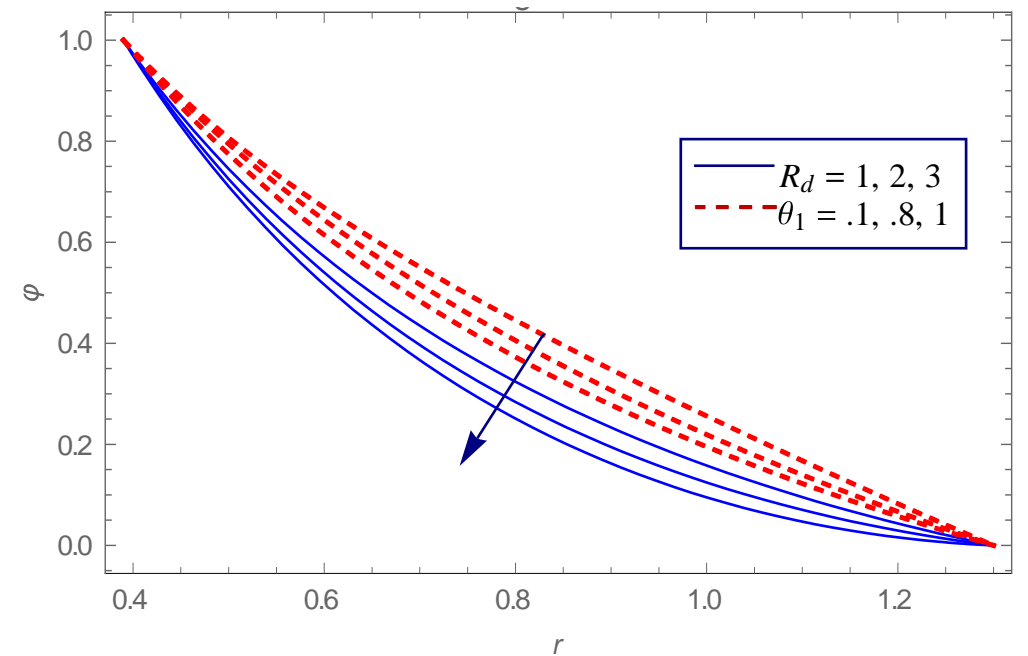

Fig. 11. The nanoparticles concentration of fluid particles $\varphi$ is plotted against $r$, for $N_{r}=.1, G_{r}=3, M=1, \lambda_{1}=.3, P_{r}=$ $.7, R_{b}=.2, E_{c}=.1, R_{d}=.4, \theta_{1}=1.1, P_{e}=1$

\subsection{Motile Microorganisms Density Profiles}

The impact of the thermophoresis parameter $N_{t}$ and the Brownian motion parameter $N_{b}$ on the density of the motile microorganisms of the fluid can be seen from Figure 12 . The boosting values of $N_{t}$ decay the density of the motile microorganisms, but the inverse impact happens for expanding $N_{b}$. Figure 13 is outlined to decipher the varieties in the density of the motile microorganisms affected by the non-linear radiation parameter $R_{d}$ and Prandtl number $P_{r}$. It is noted that It is noticed that an amplifying in $R_{d}$ and $P_{r}$ prompts a decline in the density of the motile microorganisms.

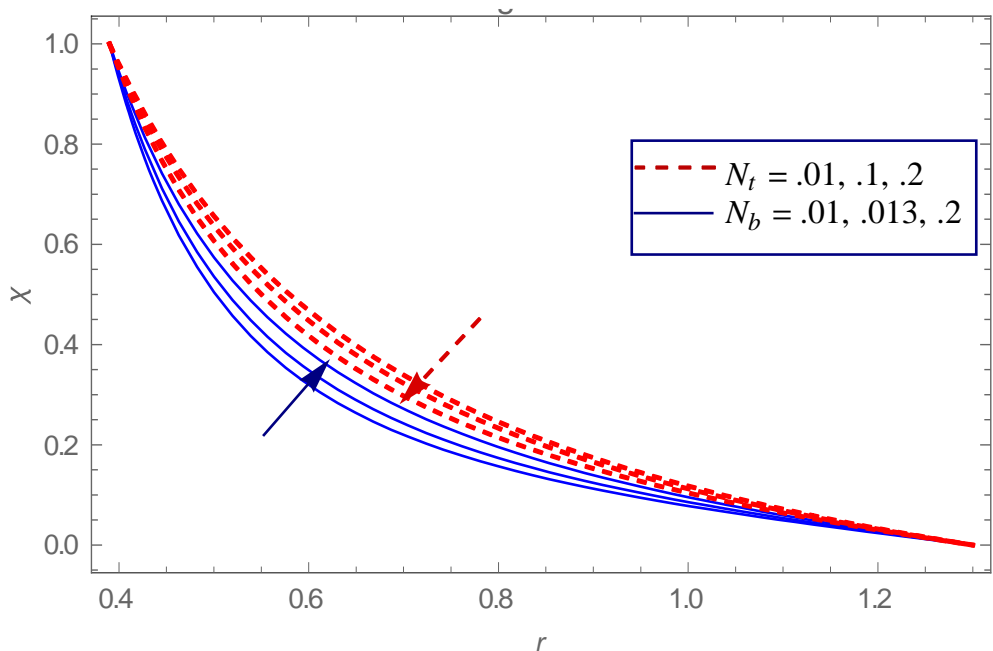

Fig. 12. The density of motile microorganisms $\chi$ is plotted against $r$, for $N_{r}=.1, G_{r}=3, M=1, \lambda_{1}=.3, P_{r}=.7, R_{b}=$ .2, $E_{c}=.1, R_{d}=.4, \theta_{1}=1.1, P_{e}=1$ 


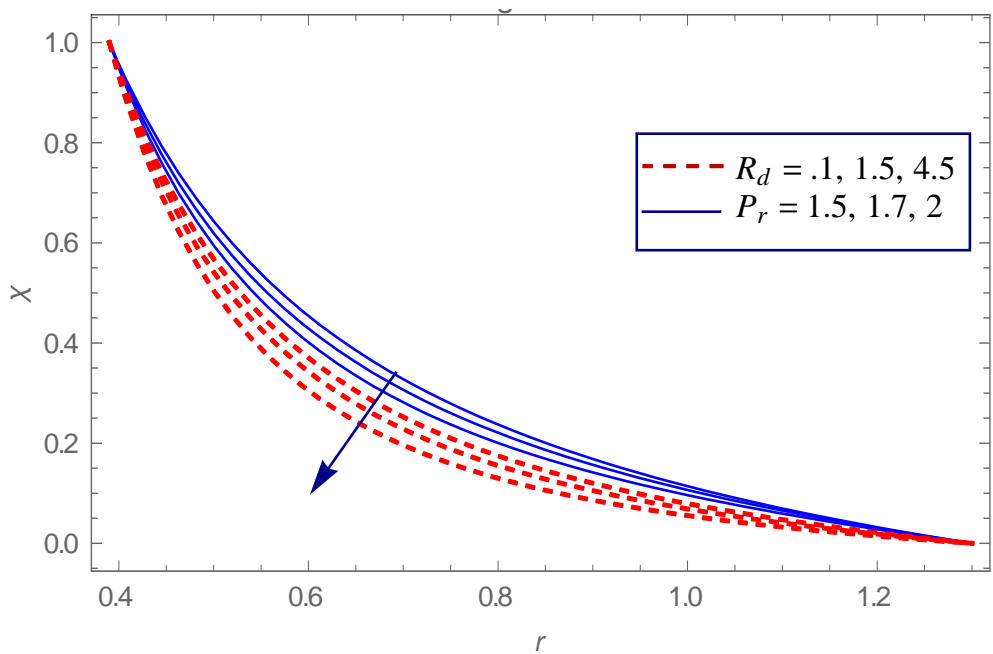

Fig. 13. The density of motile microorganisms $\chi$ is plotted against $r$, for $N_{r}=.1, G_{r}=3, M=1, \lambda_{1}=.3, P_{r}=.7, R_{b}=$ $.2, E_{c}=.1, N_{t}=.1, \theta_{1}=1.1, N_{b}=.1$

\subsection{Wall Shear Stresses (WSS)}

The effects of the physical parameters on the shear stresses are outlined in Figure 14 and 15. It is seen that raising in $\phi_{1}$ declines the shear stress in the large gap between the two tubes and the inverse impact happens in the narrow part. The effect of $\lambda_{1}$ upgrades the shear stress at the inward surface of the external cylinder, while the switch impacts are seen at the external surface of the internal cylinder.

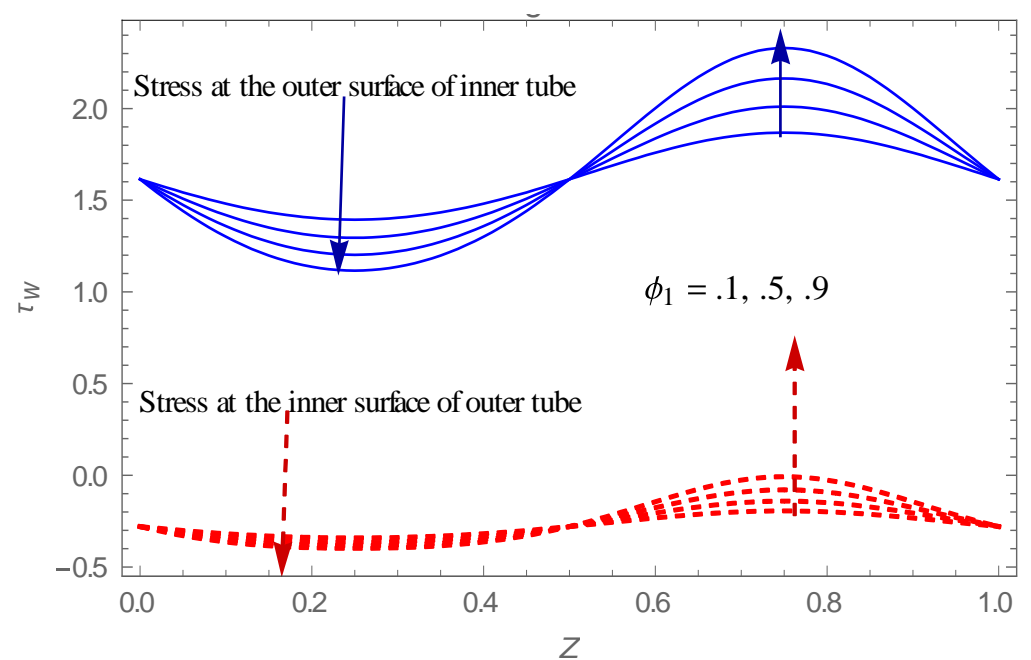

Fig. 14. The wall Shear stresses $\tau_{w}$ is plotted against $z$, for $G_{r}=$ $3, N_{b}=N_{t}=E_{c}=.1, N_{r}=.1, P_{r}=.7, R_{b}=.2, R_{d}=.4, \theta_{1}=$ $1.1, P_{e}=1, \lambda_{1}=.3$ 


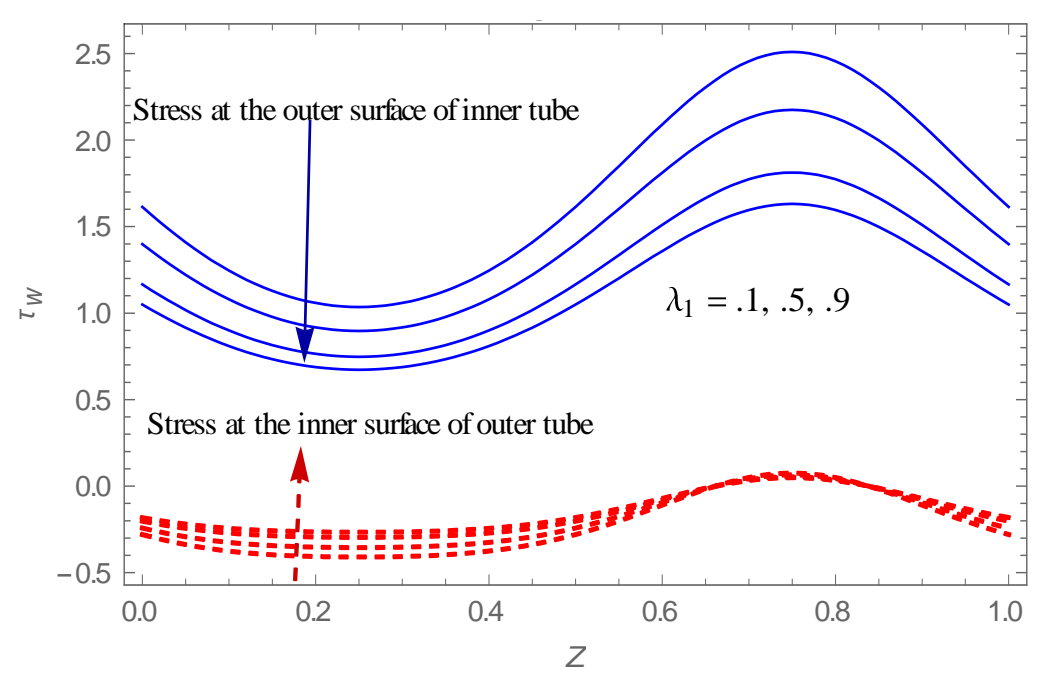

Fig. 15. The wall Shear stresses $\tau_{w}$ is plotted against $z$, for $G_{r}=$ $3, N_{b}=N_{t}=E_{c}=.1, N_{r}=.1, P_{r}=.7, R_{b}=.2, R_{d}=.4, \theta_{1}=$ $1.1, P_{e}=1$

\subsection{The Pressure Gradient}

The pressure gradient $\frac{d p}{d z}$ has been plotted in Figure 16 and 17. These figures demonstrate that the pressure gradient is little in the huge hole between the two cylinders, where it is enormous in the limited part. Figure 16 demonstrates that the pressure gradient increment by expanding the nonNewtonian parameter $\lambda_{1}$ and the magnetic parameter $M$, while Figure 17 demonstrates that the pressure gradient decline by expanding the bioconvection Rayleigh number $R_{b}$ and the thermophoresis parameter $N_{t}$.

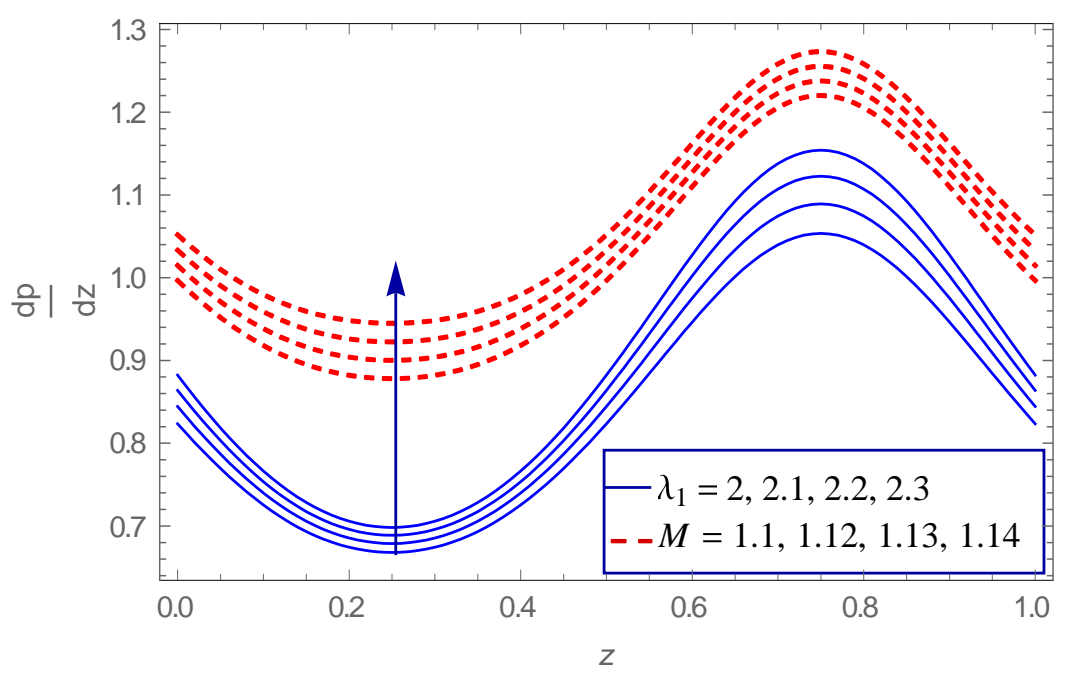

Fig. 16. The pressure gradient is plotted against $z$, for $N_{b}=N_{t}=$ $E_{c}=N_{r}=.1, R_{b}=.2, R_{d}=.4, \theta_{1}=1.1, P_{r}=.7, P_{e}=1, G_{r}=$ $3, Q=.2$ 


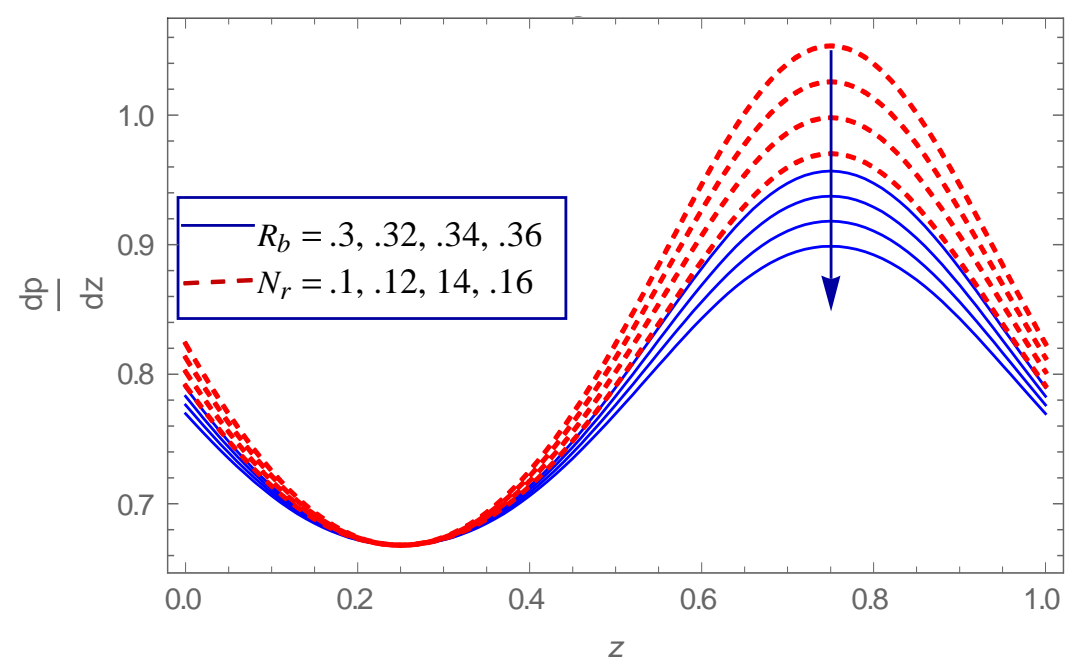

Fig. 17. The pressure gradient is plotted against $z$, for $N_{b}=N_{t}=$ $E_{c}=\lambda_{1}=2, M=1, R_{d}=.4, \theta_{1}=1.1, P_{r}=.7, P_{e}=1, G_{r}=3, Q=$ .2

\subsection{The Pressure Rise}

The impact of the physical parameters on the pressure rise $\Delta p$ versus the Grashof number $G_{r}$ are presented though Figure 18 and 19. The pressure rise increment by expanding the non-Newtonian parameter and the magnetic parameter, while it is decline by expanding increasing the bioconvection Rayleigh number and the thermophoresis parameter.

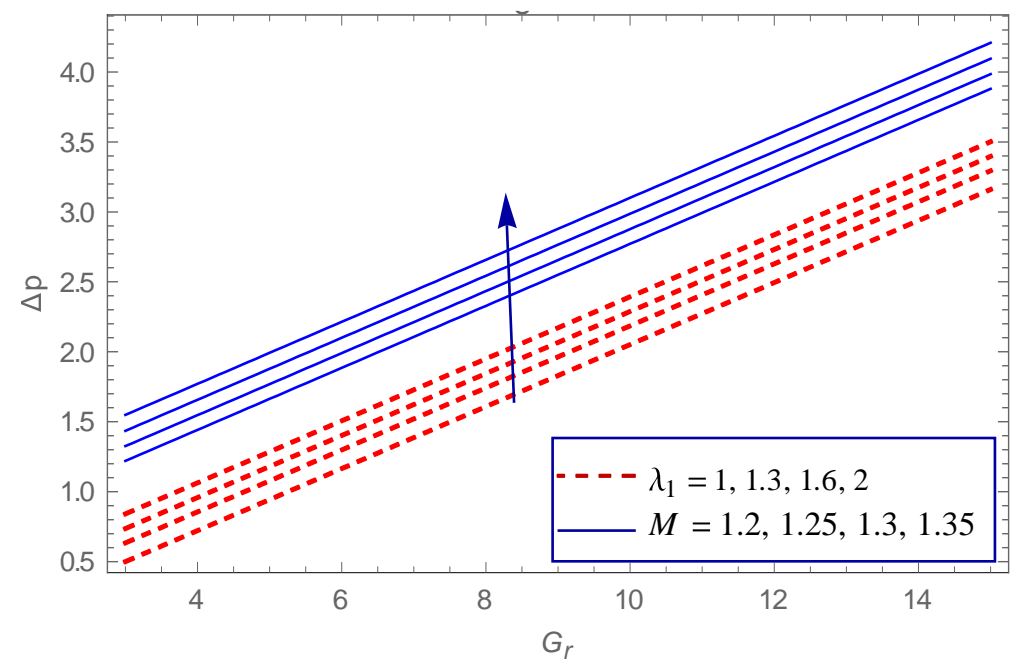

Fig. 18. The pressure rise is plotted against $G_{r}$, for $N_{b}=N_{t}=$ $E_{c}=N_{r}=.1, R_{b}=.2, R_{d}=.4, \theta_{1}=1.1, P_{r}=.7, P_{e}=1, G_{r}=$ 3 


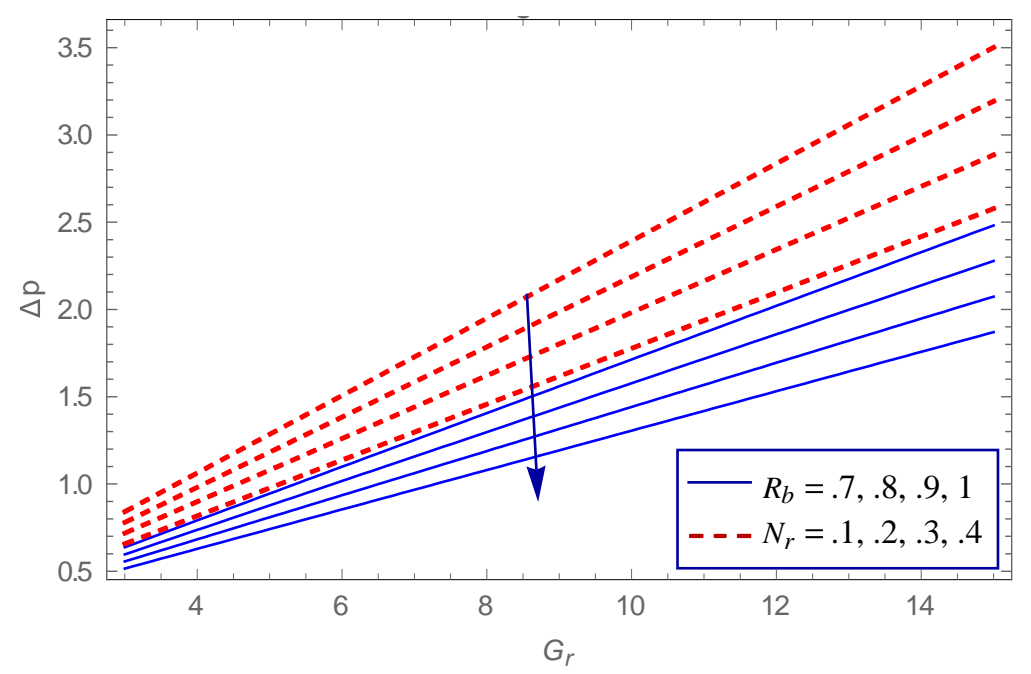

Fig. 19. The pressure rise is plotted against $G_{r}$, for $N_{b}=N_{t}=$ $E_{c}=\lambda_{1}=2, M=1, R_{d}=.4, \theta_{1}=1.1, P_{r}=.7, P_{e}=1, G_{r}=3$

\section{Conclusion}

Investigation of the peristaltic flow of Jeffrey nanofluid containing motile gyrotactic microorganisms through an endoscope under the impacts of non-linear thermal radiation, magnetic field, and viscous dissipation carried out. Our system of equations is solved numerically by using the Rung-Kutta-Merson method with Newton iteration in a shooting and matching technique. Fundamental highlights of the investigation are featured underneath

i. A relationship between courses of action of our fluid motion and by virtue of the missing of nanoparticles and microorganisms has been had under the effects of the non-Newtonian parameter $\lambda_{1}$ and the temperature ratio parameter $\theta_{1}$. As foreseen the fluid velocity show diminishing conduct with the nearness of nanoparticles and microorganisms, while the temperature profile is higher than that in the missing nanoparticles and microorganisms.

ii. The velocity exhibits more response in the case of deformable tubes than the annulus under the effect of the magnetic field.

iii. The temperature increases under the effects of the Brownian motion parameter $N_{b}$ and the thermophoresis parameter $N_{t}$.

iv. The non-linear radiation parameter and Prandtl number reveal opposite behaviour on the motile microorganisms' density.

v. An increment in the amplitude ratio $\phi_{1}$ declines the shear stress in the large gap between the two tubes and the inverse impact happen in the narrow part.

vi. Large values of the buoyancy ratio parameter $N_{r}$ and the bioconvection Rayleigh number $R_{b}$ tend to decline the pressure gradient.

vii. The greater influence of the non-Newtonian and magnetic parameters enhances the pressure rise. 


\section{References}

[1] Mekheimer, Kh S. "Peristaltic transport of a Newtonian fluid through a uniform and non-uniform annulus." Arabian Journal for Science and Engineering 30, no. 1 (2005): 69-83.

[2] Akbar, Noreen Sher, and S. Nadeem. "Peristaltic flow of a micropolar fluid with nano particles in small intestine." Applied Nanoscience 3, no. 6 (2013): 461-468.

https://doi.org/10.1007/s13204-012-0160-2

[3] Nadeem, S., Noreen Sher Akbar, T. Hayat, and Awatif A. Hendi. "Peristaltic flow of Walter's B fluid in endoscope." Applied Mathematics and Mechanics 32, no. 6 (2011): 689-700. https://doi.org/10.1007/s10483-011-1449-7

[4] Khan, Noor Saeed, Taza Gul, Muhammad Altaf Khan, Ebenezer Bonyah, and Saeed Islam. "Mixed convection in gravity-driven thin film non-Newtonian nanofluids flow with gyrotactic microorganisms." Results in physics 7 (2017): 4033-4049. https://doi.org/10.1016/i.rinp.2017.10.017

[5] Raju, C. S. K., Mohammad Mainul Hoque, and T. Sivasankar. "Radiative flow of Casson fluid over a moving wedge filled with gyrotactic microorganisms." Advanced Powder Technology 28, no. 2 (2017): 575-583. https://doi.org/10.1016/i.apt.2016.10.026

[6] Bhatti, M. M., A. Zeeshan, and R. Ellahi. "Simultaneous effects of coagulation and variable magnetic field on peristaltically induced motion of Jeffrey nanofluid containing gyrotactic microorganism." Microvascular research 110 (2017): 32-42.

https://doi.org/10.1016/j.mvr.2016.11.007

[7] Mekheimer, Kh S., Y. Abd Elmaboud, and A. I. Abdellateef. "Particulate suspension flow induced by sinusoidal peristaltic waves through eccentric cylinders: thread annular." International Journal of Biomathematics 6, no. 04 (2013): 1350026-1350051.

https://doi.org/10.1142/S1793524513500265

[8] Bhatti, Muhammad Mubashir, and Ahmed Zeeshan. "Study of variable magnetic field and endoscope on peristaltic blood flow of particle-fluid suspension through an annulus." Biomedical Engineering Letters 6, no. 4 (2016): 242249.

https://doi.org/10.1007/s13534-016-0226-2

[9] Abdelsalam, Sara I., and Muhammad Mubashir Bhatti. "The impact of impinging TiO2 nanoparticles in Prandtl nanofluid along with endoscopic and variable magnetic field effects on peristaltic blood flow." Multidiscipline Modeling in Materials and Structures 4, no. 3 (2018): 530-548.

https://doi.org/10.1108/MMMS-08-2017-0094

[10] Zeeshan, A., N. ljaz, M. M. Bhatti, and A. B. Mann. "Mathematical study of peristaltic propulsion of solid-liquid multiphase flow with a biorheological fluid as the base fluid in a duct." Chinese Journal of Physics 55, no. 4 (2017): 1596-1604. https://doi.org/10.1016/i.cjph.2017.05.020

[11] Mekheimer, Kh S. "The influence of heat transfer and magnetic field on peristaltic transport of a Newtonian fluid in a vertical annulus: application of an endoscope." Physics letters A 372, no. 10 (2008): 1657-1665. https://doi.org/10.1016/i.physleta.2007.10.028

[12] Hayat, Tasawar, Javaria Akram, Ahmed Alsaedi, and Hina Zahir. "Endoscopy and homogeneous-heterogeneous reactions in MHD radiative peristaltic activity of Ree-Eyring fluid." Results in physics 8 (2018): 481-488. https://doi.org/10.1016/i.rinp.2017.12.056

[13] Eldabe N., Ramadan S., and Awad A." Analytical and numerical treatment to study the effects of Hall currents with viscous dissipation, heat absorption and chemical reaction on peristaltic flow of Carreau nanofluid." Thermal Science (2019).

https://doi.org/10.2298/TSCI190319309N

[14] Ellahi, R., and F. Hussain. "Simultaneous effects of MHD and partial slip on peristaltic flow of Jeffery fluid in a rectangular duct." Journal of Magnetism and Magnetic Materials 393 (2015): 284-292.

https://doi.org/10.1016/i.jmmm.2015.05.071

[15] Mekheimer, Kh S. "Peristaltic transport of a particle-fluid suspension through a uniform and non-uniform annulus." Applied Bionics and Biomechanics 5, no. 2 (2008): 47-57.

https://doi.org/10.1080/11762320802376183

[16] Hasona, W. M., A. A. El-Shekhipy, and M. G. Ibrahim. "Combined effects of magnetohydrodynamic and temperature dependent viscosity on peristaltic flow of Jeffrey nanofluid through a porous medium: Applications to oil refinement." International Journal of Heat and Mass Transfer 126 (2018): 700-714.

https://doi.org/10.1016/j.ijheatmasstransfer.2018.05.087 
[17] Rachid, H., and M. T. Ouazzani. "Electro-magnetohydrodynamic Peristaltic Pumping of a Biviscosity Fluid Between Two Coaxial Deformable Tubes Through a Porous Medium." ACTA PHYSICA POLONICA B 48, no. 9 (2017): 15151527.

https://doi.org/10.5506/APhysPolB.48.1515

[18] Wahid, Nur Syahirah, Mohd Ezad Hafidz Hafidzuddin, Norihan Md Arifin, Mustafa Turkyilmazoglu, and Nor Aliza Abd Rahmin. "Magnetohydrodynamic (MHD) Slip Darcy Flow of Viscoelastic Fluid Over A Stretching Sheet and Heat Transfer with Thermal Radiation and Viscous Dissipation." CFD Letters 12, no. 1 (2020): 1-12.

[19] Eldabe, Nabil T., Kawther A. Kamel, Shaimaa F. Ramadan, and Rabab Ahmed Saad. "Peristaltic Motion of EyringPowell Nano Fluid with Couple Stresses and Heat and Mass Transfer Through a Porous Media Under the Effect of Magnetic Field Inside Asymmetric Vertical Channel." Journal of Advanced Research in Fluid Mechanics and Thermal Sciences 68, no. 2 (2020): 58-71.

https://doi.org/10.37934/arfmts.68.2.5871 